\title{
Engineering Investigations of Dufour and Soret Effect on MHD Heat and Mass Transfer with Radiative Heat Flux in a Liquid over a Rotating Dick.
}

\author{
Muhammad Ismail Mohmand ${ }^{1 *}$, Mustafa Bin Mamat', Qayyum Shah ${ }^{2,3}$ \\ ${ }^{1,2,3}$ Faculty of Informatics and Computing, Universiti Sultan Zainal Abidin, UniSZA, Malaysia. \\ *Corresponding Author Email: muhammadismail1745@gmail.com
}

\begin{abstract}
:
This paper deals with the examination effect of Dufour and Soret Effect on MHD heat and mass transfer with radiative heat flux in a liquid over a rotating dick. The problem is solved by HAM (Homotopy Analysis Method). The arrangement of Non-Linear Partial Differential Equations (PDEs) which administer the stream, heat and mass exchange qualities is changed into Ordinary Differential Equations (ODEs). It is discovered from the current exploration that Dufour and radiation impacts cause diminishments in the liquid temperature. The effect of suction diminishes the speed, temperature, what's more, focus profiles essentially in the limit layer.
\end{abstract}

Keywords: Dufour effect, Soret Effect, MHD, Heat and Mass Transfer, Rotating Disc.

\section{Introduction}

Dufour and Soret impacts on MHD heat and mass exchange with radioactive warmth transition in a moment review fluid over a pivoting circle has been examined by researchers throughout the years with an end goal to decide, conditions that would foresee the development of MHD liquids in a turning way heading. Its application has been fruitful in the bloodstream and blood transfusion and additionally mechanical advancements that exchange fluids, replicating this rotational movement (Pal et al., 2016).

Free convection stream frequently happens in nature in light of temperature contrast and furthermore fixation distinction. Regular convection is essential in an industrialized process for the outline of reliable hardware, for control plant, space vehicles, burning turbines and different impetus gadgets for airships. Shah et al. (2017) dissected numerically insecure free convection warmth and mass exchange over a walters-B viscoelastic heat past a semi unbounded vertical plate. Reddy et al, (2017) completed the numerical investigation of free convection stream past an upright cone with variable warmth and mass motion. Muthuraj et al (2017) talked about the compound responses impacts on unsteady free convective and mass exchange stream on a vertical cone by warm age/retention in the nearness of variable divider temperature or variable

Divider focus. Hayat et al. (2017) talked about the impacts of substance response and radiation on MHD free convection stream with thick scattering on unexpectedly began sizeable vertical plate. Sivaraj et a1.(2016) considered the variable electric conductivity and concoction response impacts on free convection stream over an upright cone. Raju et al. (2017) contemplated the implications of substance response and warmth age/retention on free convective spill out of a vertical cone in nearness of uniform divider temperature/uniform divider focus.

Viscous spread portrays the degeneration of mechanical essentialness into the warm vitality. Such wonder occurs in all the stream systems. Be that as it may, for different stream setups, the characteristics of gooey dispersal are routinely expelled. It is basic just for the structures having more basic speed and speed points independently. It is thusly that the gooey scattering is exhibited in the present examination. Synchronous characteristics of gooey dispersal, Dufour-Soret, and thermophore sis in a radioactive thick material stream towards isothermal wedge are explored by Pal and Mondal (2013). Zaib and Shafie (2014) examined thick dispersing edge in artificially reacting stratified expanded stream of gooey liquid considering Hall current. Hayat et al. (2015) inspected the reasonability of thick dispersal and MHD impacts considering Jeffrey and second-grade liquid models. A three-dimensional dissipative broadened stream of radioactive Powell-Eyeing nonmaterial is tended to by Mahanthesh et al. (2017)

The Soret and Dufour impacts are basic in higher temperature and center slants. Ramzan et al. (2017) discussed the Soret and Dufour impacts by common convection from a vertical truncated cone absorbed a permeable medium with variable divider temperature or variable divider obsession. The impacts of Soret and Dufour on customary convection stream on an upright cone in a permeable medium by reliable divider warmth and mass movements have been considered by Pal and Mondal (2013). Hayat et al. (2017) analyzed the variable thickness to consider Soret and Dufour results for specific convection course through a vertical surface inside a penetrable medium.

Moody et al. (2014) considered the Soret and Dufour impacts on non Darcian standard convection spill out of a vertical wavy surface enveloped by a penetrable medium. Zaib and Shafie (2014) considered blend reaction to examine Soret and Dufour 
results for hydromagnetic free convective stream past an unbounded vertical vulnerable plate. Ashraf et al. (2015).

Influenced by the examinations and applications specified over, the focal topic of the present work is to investigate the direct of an unpredictable thick fluid influenced by Dufour and Soret impacts with warmth and mass trade. Execution of HAM prompts centered plan (Hayat et al., 2016). The creating varying parameters are plotted for specific regards to research their impact on the speed and warm fields. The estimations of surface drag power and warmth conversion scale are numerically clarified. The regulating conditions are poor around HAM, and for authenticity, the results are differentiated and numerical BVP4C package. In the going with range, the issue is characterized, bankrupt down and inspected

$$
\begin{aligned}
& \frac{\partial u}{\partial r}+\frac{\partial w}{\partial x}+\frac{u}{r}=0, \\
& \rho\left(\frac{\partial u}{\partial t}+u \frac{\partial w}{\partial z}+w \frac{\partial u}{\partial z}-\frac{v^{2}}{r}\right)=\frac{-\partial p}{\partial r}+\mu\left(\frac{\partial^{2} u}{\partial r^{2}}+\frac{\partial^{2} u}{\partial z^{2}}+\frac{1}{r} \frac{\partial u}{\partial r}-\frac{u}{r^{2}}\right)
\end{aligned}
$$

\section{Definition of the Problem}

The issue is exhibited by the properties of liquid characterized by Dufour and Soret impacts; the fundamental problem is that of the nonstraight or temperamental stream of fluids. Dufour impacts demonstrate how molecule development influences temperature changes, while then again Soret impact responds these. Soret impact illustrates how temperature changes affect molecule development.

These impacts affect liquids conducted in rotational movement To solve this challenge, MHD liquids are utilized. These are electrically leading fluids, whose tradition and rate of exchange of mass is gooey and incompressible within sight of warm radiation and warm dispersion

\subsection{Control}

A terminal plate is utilized, to adjust the traditions of hydrodynamics. This is the behavioral components of liquid, fundamentally laminar and turbulent stream exhibited and dictated by speed and course of the flow.

To control the composition of the stream in thermodynamics, an attractive motion is utilized to impact flow, speed, and heading of the stream of MHD liquids. These liquids distort keep up their properties of the room, under the attractive motion and thus kept up in their way of the flow. The degree to which these is conceivable is dictated by mathematical figuring's, which have been contrived since the issue was first recognized

\subsection{Homotopy Analysis Method (HAM) Solution}

TheHomotopy Analysis Method (HAM) gives arrangement of obscure functions. The union of this strategy relies upon the best possible choice of the assistant capacities and starting theories. The arrangement type of anonymous functions

$$
\begin{aligned}
& f_{n} \mid z=\sum_{n=0}^{\infty} \sum_{n=0}^{\infty} \alpha_{m=x^{2}}^{*} \exp (-n z), \\
& g_{n}|z|=\sum_{n=0}^{\infty} \sum_{n=0}^{\bar{n}} b_{m, t^{2}} x^{k} \exp (-n z) .
\end{aligned}
$$

$$
\begin{aligned}
& g_{n}|t|=g_{n}+c_{4}+c_{2} e^{-1} \\
& p_{n}|z|=p_{m}^{b}+c_{n} \\
& \theta_{m}|x|=\theta_{m}+c_{1}+c_{9} e^{-4} \\
& \phi_{n}|z|=\phi_{m}^{2}+c_{n}+c_{10} e^{*} \\
& \text { Where } f^{4}, g^{4}, p^{4}, \theta^{4}, \wedge \phi^{6} \text { arethe special solutions }
\end{aligned}
$$

\subsection{Determination}

Since MHD liquids keep up the properties of the room, differential Equations have been connectedto decide the point ofconfinement conditions of Dufour and Soret impact passable for a fluid to keep up way in a round tube.

\section{Comparison, Results, and Discussion}

Homotopy Analysis Method Equation is a semi expository strategy whereby one capacity is persistently misshaping into another (He, 2003). Inthis count; capacities, substantial amounts are identified with subordinates, the rate of progress: the capabilities here being temperature and mass fixation. Different aides as weight, which is the constrained convection because of attractive transition, grinding and gravity, which is a natural convection, are viewed as in respect to the principal subsidiaries which are; mass fixation slope and temperature inclination. These two subordinates are controlled in the Dufour and Soret impacts on MHO warmth and mass exchange with radioactive warmth transition in a moment review fluid over a pivoting plate test. It is accepted that gravity impacts are missing in this examination.

The fractional differential Equations of the congruity Equation are lessened to ordinary differential Equation where one independent factor is landed at. This esteem is connected to decide the entire states at substantial conditions.

Figures 1(a\}- (d) exclusively depict the assortments of the winding velocity fragment $\mathrm{f}$ for different estimations of relentless quality parameter $\mathrm{S}$ and suction parameter A. From figs. 1(a), (c) and (d), it can be seen that the extended part $\mathrm{f}$ lessens with an extension in the unwavering quality parameter. With growing suction parameter an, a greater amount of the inflowing fluid passes clearly into the porous divider. Thusly, when suction is associated with the circle surface, the winding rate parts lessen rapidly with the extending suction parameter, as showed up in fig. 1(b). Furthermore it can be seen that for more suction the winding velocity is nearly nothing. Figures $1(a)$ - (c) exhibit the center speed profile $f$ with different stream parameters
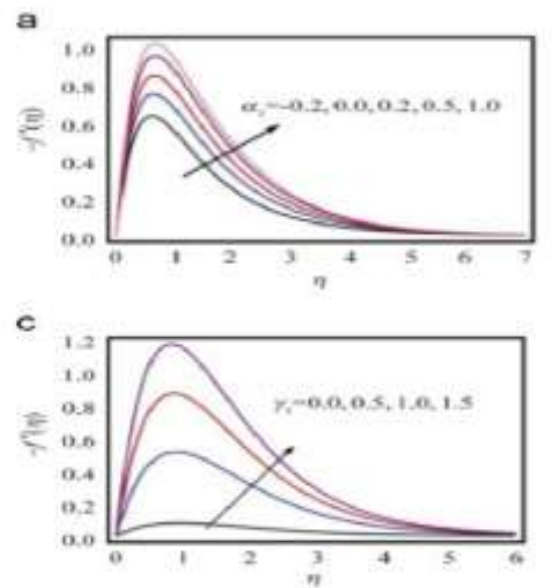

From figures 2(a) - (c) it can be watched that the center point portion $\mathrm{f}$ is reducing when the determination parameter increases. This is a direct 
result of the way that the breaking point layer near the surface of the circle is hurled outward in view of the emanating power procedures in all directions
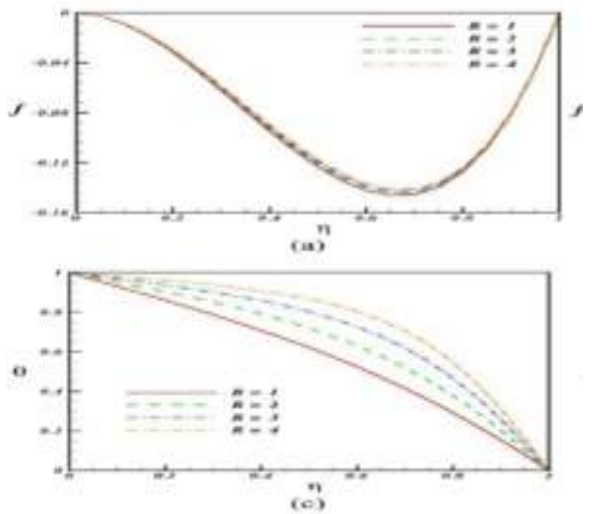

b

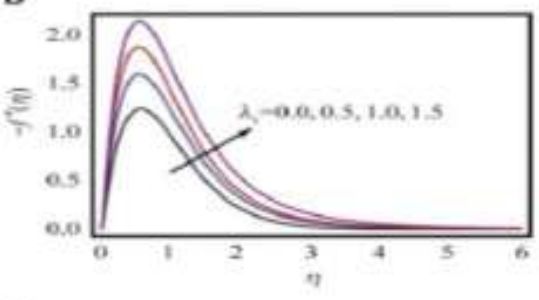

d

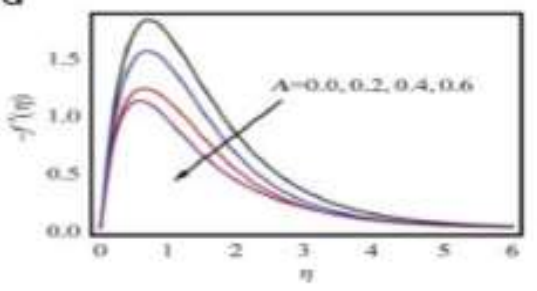

b

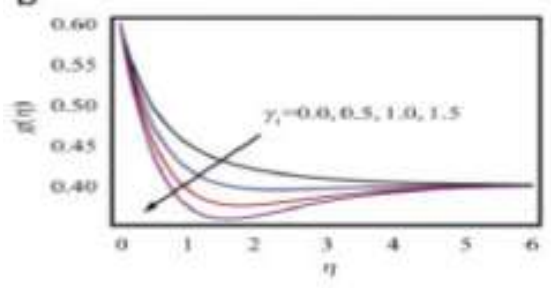

d

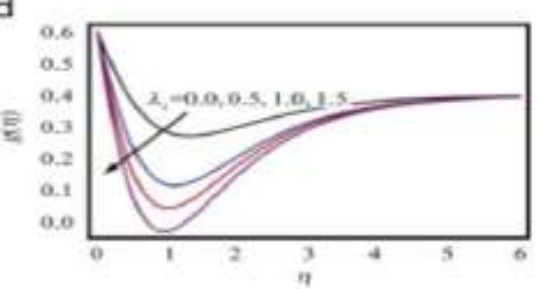

a

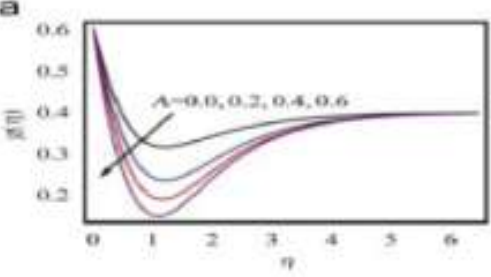

c

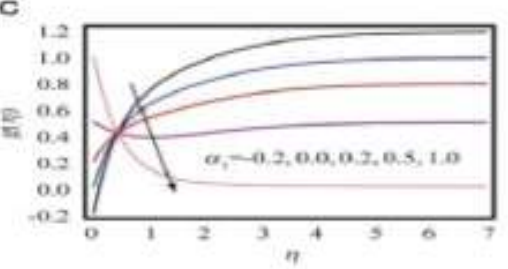

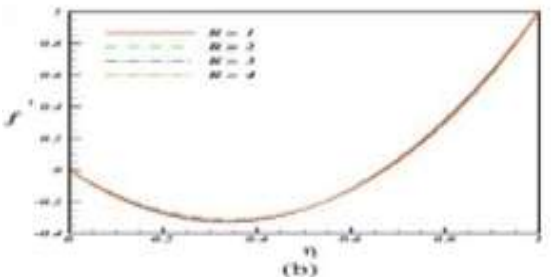

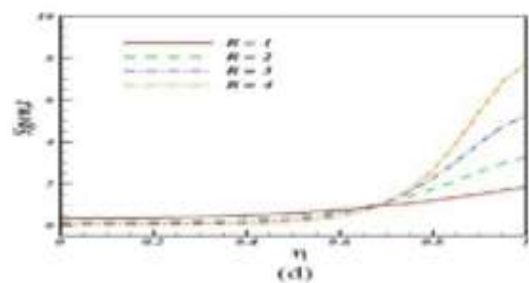

In figs. 3(a) - (c) we have plotted the azimuthal fragment of speed $g$ for dauntlessness parameter $S$. The effect of predictable quality parameter $S$ on the azimuthal speed profile is showed up in figs. 3(a) - (c), where it can be watched that for a broad estimation of $\mathrm{S}$, the azimuthal speed section is at its most noteworthy

\section{Conclusion}

The analysis above explained, aids us to conclude that thermodynamics flow in MHD fluids is dependent on Soret and Dufour effects. These conclusion impacts on the rotating disc experiment and tabulation of variables applied in the determination of limit states for MHD fluids in rotational motion.

The experiments deduced from these, and other diverse innovation based on Dufour and Sorets stresses have aided in analyzing situations to form statistical data. This data is used to map behavior and make predictions of the outcome of MHD behavior under valid variables and circumstances.

\section{References}

[1] Ashraf, M. B., Hayat, T., Shehzad, S. A., \& Ahmed, B. (2017). Thermophoresis and MHD mixed convection three-dimensional flow of viscoelastic fluid with Soret and Dufour effects. Neural Computing and Applications, 1-13. Dufour and Soret effects on MHD available at https:/!link.springer.com/

[2] Hayat, T., Khan, M. 1., Farooq, M., Gull, N., \&Alsaedi, A (2016). Unsteady three-dimensional mixed convection flow with variable viscosity and thermal conductivity. Journal of Molecular Liquids, 223,1297-1310.

[3] Hayat, T., Khan, M. 1., Waqas, M., \&Alsaedi, A. (2017). Newtonian heating effect in nanofluid flow by a permeable cylinder. Results in Physics, 7, 256-262.

[4] Hayat, T., Ullah, 1., Muhammad, T., \&Alsaedi, A. (2017). Radiative three-dimensional flow with Soret and Dufour effects. International Journal of Mechanical Sciences.

[5] Hayat, T., Waqas, M., Shehzad, S. A., \&Alsaedi, A. (2015). MHD stagnation point flow of Jeffrey fluid by a radially stretching surface with viscous dissipation and Joule heating. Journal of Hydrology and Hydromechanics, 63(4),311.

[6] Klinger, J. M. (2015). On the Rare Earth Frontier.University of California, Berkeley.

[7] Loper, D. E., \& Benton, E. R. (1970). On the spin-up of an electrically conducting fluid Part 2. Hydromagnetic spin-up between infinite flat insulating plates. Journal of Fluid Mechanics, 43(4), 785-799.

[8] Mahanthesh, B., Gireesha, B. J., \& Gorla, R. S. R. (2017). Unsteady three-dimensional MHD flow of a nanoEyring-Powell fluid past a convectively heated stretching sheet in the presence of thermal radiation, viscous dissipation and Joule heating. Journal of the Association of Arab Universities for Basic and Applied Sciences, 23, 75-84. 